\title{
Usage of drug-coated balloon in acute ST-segment elevation myocardial infarction?!
}

\author{
Boris Starčević*, Mario Sičaja, Ilko Vuksanović, Dražen Šebetić, Miroslav Raguž \\ University Hospital Dubrava, Zagreb, Croatia
}

Novel balloons coated with paclitaxel (drug-coated balloon, DCB) were recently introduced in Croatia. Their usual indication is the treatment of in-stent restenosis and small vessels disease with significant stenosis.

We describe a case of usage of DCB in acute STEMI after thrombus aspiration. A 46-year old male, with a history of alcohol abuse, presented with acute anteroseptal STEMI. An emergency coronarography was performed and culprit lesion was found in mid LAD. After thrombus aspiration a DCB PTCA LAD was performed with optimal angio appearance. His clinical course was uneventful.

The paclitaxel DCB is an emerging device in percutaneous coronary intervention. Recently, this technology has rapidly evolved with newer studies which assess the value of DCB in coronary applications other than in-stent restenosis. In our oppinion, DCB can be safely used in selected cases where stent implantation could be associated with increased risk of complications (i.e. increased risk of Gl bleeding).

KEYWORDS: coronary artery disease, drug-coated balloon, ST-segment elevation myocardial infarction, percutaneous coronary intervention.

Received: $5^{\text {th }}$ Mar 2013

*Address for correspondence: Klinička bolnica Dubrava, Avenija Gojka Šuška 6 , HR-10000 Zagreb, Croatia.

Phone: $+385-1-2902-444$

E-mail: starki_pl@yahoo.com

\section{Literature}

1. Wöhrle J. Drug-coated balloons for coronary and peripheral interventional procedures. Curr Cardiol Rep. 2012;14(5):635-41.

2. Belkacemi A, Agostoni P, Nathoe HM, et al. First results of the DEB-AMI (drug eluting balloon in acute ST-segment elevation myocardial infarction) trial: a multicenter randomized comparison of drug-eluting balloon plus bare-metal stent versus bare-metal stent versus drug-eluting stent in primary percutaneous coronary intervention with 6-month angiographic, intravascular, functional, and clinical outcomes. J Am Coll Cardiol. 2012;59(25):2327-37.

3. Navarese EP, Austin D, Gurbel PA, et al. Drug-coated balloons in treatment of in-stent restenosis: a meta-analysis of randomised controlled trials. Clin Res Cardiol. 2012. Dec 20. [Epub ahead of print].

4. Loh JP, Waksman R. Paclitaxel drug-coated balloons: a review of current status and emerging applications in native coronary artery de novo lesions. JACC Cardiovasc Interv. 2012;5(10):1001-12. 